\title{
A Subnanosecond Pulsed Electric Field System for Studying Cells Electropermeabilization
}

\begin{abstract}
This article presents an experimental arrangement which, using 3-D numerical modeling, aims to study biomedical effects using subnanosecond pulsed electric fields (PEFs). As part of a major effort into developing contactless technology, the final aim of this study is to determine conditions of the applied PEFs (number of pulses, strength, pulse repetition frequency) able to produce electropermeabilization. The arrangement uses a pulsed power generator producing voltage impulses with an amplitude of up to $20 \mathrm{kV}$ on a $50-\Omega$ matched load, with a rise time of $100 \mathrm{ps}$ and a duration of $600 \mathrm{ps}$. During the preliminary study reported here, samples containing $E$. Coli were exposed to PEFs in a 4-mm standard electroporation cuvette, allowing the application of a peak electric field strength of up to $60 \mathrm{kV} / \mathrm{cm}$. The studies were facilitated by detailed 3-D electromagnetic modeling of the electric field distribution generated by voltage impulses inside the system. Due to the nature of tests, the numerical analysis played an essential role in the interpretation of results. Preliminary biological results reported in this study are very encouraging, showing that trains of 5000 to $\mathbf{5 0 0 0 0}$ pulses applied at a pulsed repetition frequency of $200 \mathrm{~Hz}$ (maximum PRF) can efficiently induce $\boldsymbol{E}$. Coli electropermeabilization.
\end{abstract}

Index Terms-Biological cells, electropermeabilization, pulsed electric fields (PEFs), pulsed power, subnanosecond pulses.

Manuscript received August 28, 2020; revised September 23, 2020 an September 25, 2020; accepted October 24, 2020. This work was supported by the Agence Nationale de la Recherche (ANR) under Contract ANR18-ASTR-0019. The review of this article was arranged by Senior Editor R. P. Joshi. (Corresponding author: Laurent Pécastaing.)

Njomza Ibrahimi, Laurent Ariztia, Marc Rivaletto, Antoine Silvestre de Ferron, and Laurent Pécastaing are with the Laboratoire des Sciences de l'Ingénieur Appliquées à la Mécanique et au Génie Électrique - Fédération IPRA, Université de Pau et des Pays de l'Adour/E2S UPPA, 64000 Pau, France (e-mail: laurent.pecastaing@univ-pau.fr).

Leslie Vallet, Franck M. Andre, and Lluis M. Mir are with Université ParisSaclay, Institut Gustave Roussy, CNRS, Metabolic and Systemic Aspects of Oncogenesis (METSY), 94805 Villejuif, France (e-mail: luis.mir@cnrs.fr).

Bucur Mircea Novac is with the Wolfson School of Mechanical, Electrical and Manufacturing Engineering, Loughborough University, Loughborough LE11 3TU, U.K., and also with the Laboratoire des Sciences de l'Ingénieur Appliquées à la Mécanique et au Génie Électrique - Fédération IPRA, Université de Pau et des Pays de l'Adour/E2S UPPA, 64000 Pau, France (e-mail: b.m.novac@lboro.ac.uk).

Color versions of one or more of the figures in this article are available online at http://ieeexplore.iee.org.

Digital Object Identifier 10.1109/TPS.2020.3034286 and short duration. The pore formation generates openings in the cell membrane, allowing for the transfer of therapeutic molecules across the cell membrane [2], [3]. Thus, the electroporation phenomenon contributes to the efficiency of electrochemotherapy, when used with drugs [4] and electrogenetherapy, when used with DNA plasmids [5].

PEFs can cause reversible electroporation, i.e., a temporary permeabilization of the cell membrane [6], but they can also produce irreversible electroporation, i.e., cell death, for eradicating cancerous or other unwanted cells [7].

There are basically two types of PEF techniques applied in vivo: invasive, where metallic pairs of penetrating electrodes are used to apply the electric field and noninvasive, where the electric field is generated through electrodes brought in contact with the skin, without penetration of the body. In addition, the contactless term refers here to the approach dealing with the generation of electric fields remotely from the metallic structure of a PEF source (antenna).

The electrode-based contact technology is already successfully applied in the treatment of various cancers including, but not limited to, kidney, liver, lung, and bone cancers [8]. Pulses having hundreds of ns duration applied for cancer treatment are still under clinical trials and as such are not part of regular treatments. PEFs of about $100 \mu$ s are widely used, depending on the type and the evolution of cancer while ms pulses are not used for cancer treatment. The outcome effect depends not only on the magnitude and duration of the PEFs delivered, but also on the pulse repetition frequency (PRF) and on the characteristics of the tissue, in particular, its conductivity. Irreversible electroporation using microsecond very high intensity PEFs in the tissues is widely considered in many hospitals. An advantage of this technique is that the thermal effects are considerably lower, when compared to other ablation techniques [9]. Cancer treatment techniques, which include but are not limited to chemotherapy and surgery, are unfortunately accompanied by adverse side effects imposed on the patient [10], [11]. The future of the PEF-based cancer treatment techniques is therefore closely related to the identification of new ways to avoid the challenges imposed by the existing invasive surgical procedures. The pathway toward a contactless technology, a nonsurgical procedure, which aims at overcoming some of the issues mentioned above, was studied in very few research laboratories worldwide. The reason for the very limited number of publications published in this domain is perhaps due to the fact that, depending on their output voltage, the highly specialized HV subnanosecond pulsed 
power generators required are either extremely expensive or only available at military related research centers. Even more difficult, the focusing of electromagnetic radiation with very high precision in a volume around $1 \mathrm{~cm}^{3}$, or preferably even lower, is possible only using highly sophisticated impulse antenna techniques.

Two possible solutions were suggested theoretically by Carl Baum, as part of a major research effort undertook by the Frank Reidy Research Center for Bioelectrics at Old Dominion University (USA): an impulse radiating antenna (IRA) operated in air [12], [13], in combination with a complex many layer dielectric lens [14] and another prolate-spheroidal reflector, operated underwater [15]. More recently, at the same research center, a dielectric rod antenna was also suggested as a candidate for generating subnanosecond PEFs for the stimulation of neurological tissue [16]. All the three techniques have been theoretically investigated using CST software [15]-[17], with detailed numerical studies being reported. In contrast, very limited experimental work has been published, and only using rather low voltage subnanosecond pulsed power generators, with the resulting PEFs having extremely low peak values when compared with the values required for electroporation [18]-[20], the latter work representing an effort made at University of Limoges (France). For stimulating deep inside a tissue, say at $8 \mathrm{~cm}$ [17] using intense PEFs of some tens of $\mathrm{kV} / \mathrm{cm}$, published work suggest a subnanosecond pulsed power generator with a peak output voltage in excess of $250 \mathrm{kV}$ is required [17]. Such a generator is technically extremely challenging.

Another aspect is that when using such innovative contactless delivery systems, the characteristics of the subnanosecond PEFs are different when compared with those of the existing PEF processing. A logical step is therefore to understand their action on cells by firstly using standard electrode-based PEFs driven by subnanosecond pulsed power generators. Results published by the Old Dominion team and also by a research group at Chongqing University (China), demonstrated that subnanosecond PEFs can indeed produce: permeabilization [21] followed by the transfer of molecules inside the cell [22], cell stimulation with calcium mobilization (through VGCCs activation) [23], and apoptosis (cell death) [24]-[27]. The main issue with these results however is that they suggest a very low efficiency of processing for a PEF having a relatively low peak value, say around $25 \mathrm{kV} / \mathrm{cm}$, as it is expected to be generated in a deep tissue by a contactless reflector-based system. Under such conditions, to obtain the effect on a significant percentage of cells, it was necessary to apply an extremely large number of pulses, of the order of a few millions [22].

The development of contactless PEF technologies has been a major research theme in the long history of an ongoing collaboration between Pau University and Loughborough University and two major works have been already published discussing a novel technique for food processing [28] and a subnanosecond driven prolate antenna operated underwater [29]. The latter work reports the first practical demonstration of generating underwater a peak PEF of $50 \mathrm{kV} / \mathrm{cm}$ at $8 \mathrm{~cm}$ away from any metallic structure, i.e., fulfilling the conditions required for contactless deep tissue permeabilization [17].

The arrangement reported here is using a standard electrode-based approach. In the preliminary phase of this study, it was decided to apply PEFs on the E. Coli DH5a bacteria, which are very small cells with a diameter in the range of $1 \mu \mathrm{m}$.

First, the pulsed power generator will be introduced, together with its corresponding diagnostics.

Second, a detailed 3-D model is presented, based on the CST Microwave Studio, a 3-D finite integral time-domain (FITD) electromagnetic simulation software [30]. As the direct measurement of the PEF generated inside a cuvette containing the cell culture is technically challenging, a comprehensive 3-D modeling and numerical electromagnetic analysis were essential during the experimental studies.

Third, we report encouraging preliminary results that demonstrate that a high percentage of permeabilized bacteria can be achieved using the described arrangement.

This conclusion ends with conclusions and a brief presentation of the way ahead.

\section{EXPERIMENTAL ARRANGEMENT}

For the present studies, a pulsed power generator similar to that described in [31] was adapted for use as a subnanosecond transient source capable of producing high voltage pulses with an amplitude of up to $20 \mathrm{kV}$ and having a 100-ps rise time on a $50-\Omega$ matched load, with a duration of about 600 ps. The generator is connected to the PEF processing load through a $2.5-\mathrm{m}-$ long RG214-U type coaxial $50-\Omega$ cable, having an insulator made of polyethylene with relative permittivity of 2.26. A simplified scheme of the pulsed power arrangement is shown in Fig. 1. The generator is constructed as a fast pulse forming line, with the closing switch operated under high-pressure hydrogen. To obtain a $20-\mathrm{kV}$ peak voltage output, the product of the hydrogen pressure and the gap distance of the electrode system was held to $22 \mathrm{bar} \cdot \mathrm{mm}$. This value was then adjusted to obtain other values for the output peak voltage. The output voltage is measured using a capacitive voltage divider, the main voltage probe, attached to the RG214-U coaxial cable [Fig. 1(b)]. The transmission coefficient for this probe is equal to $-46 \mathrm{~dB}$ all over the frequency bandwidth and the reflection coefficient is lower than $-12 \mathrm{~dB}$ while the cut-off frequency is close to $6 \mathrm{GHz}$.

The PEF processing load connected at the end of the RG214-U coaxial cable [Fig. 1(b)] consists of two parallel-plate electrodes made of copper. The distance between copper electrodes is fixed to $12 \mathrm{~mm}$ to allow mounting between them a standard electroporation cuvette, having a gap distance of $4 \mathrm{~mm}$ between its internal aluminum electrodes. The volume of the sample which contains the deionized water to be tested was kept at $100 \mu \mathrm{L}$ for two reasons. First, to obtain the desired PEF in water in terms of amplitude, rise time, and duration and second to reduce the impedance mismatch. To avoid any possible electric breakdown, the upper part of the cuvette was additionally filled with $1.6 \mathrm{~mL}$ of paraffin oil, having a relative permittivity 2.33 . It is important 


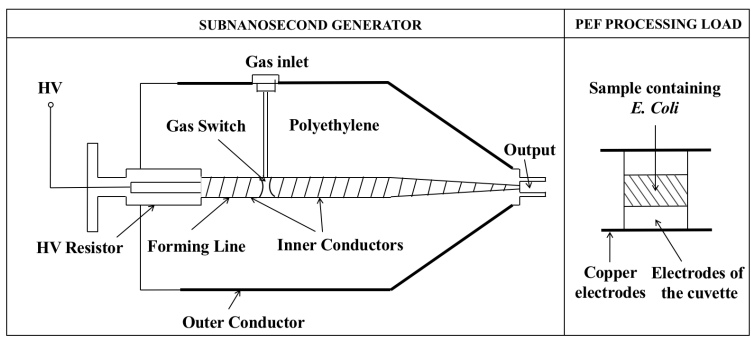

a)

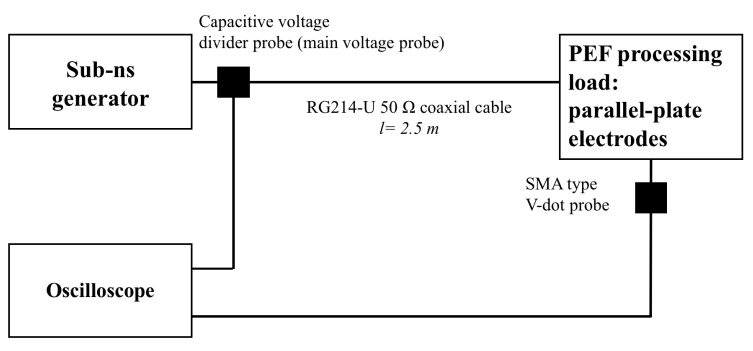

b)

Fig. 1. Pulsed power arrangement for subnanosecond PEF. (a) Subnanosecond generator (adapted after [31]) and PEF processing load. (b) Overall schematic.

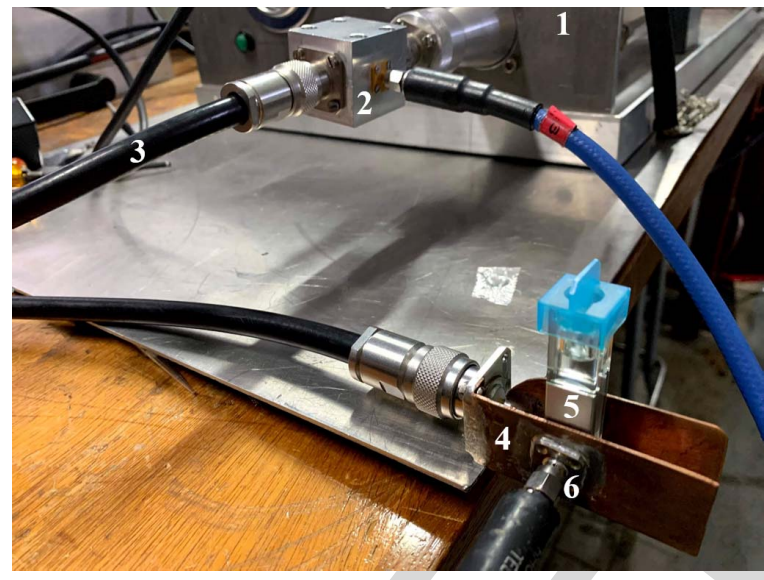

Fig. 2. Experimental arrangement for subnanosecond PEF processing. The numbers 1-6 represent the generator (1), capacitive voltage divider (main voltage probe) (2), RG214-U $50 \Omega$ coaxial cable (3), two parallel-plate electrodes (4), 4-mm electroporation cuvette (5), and SMA-type V-dot probe (6).

to note that the dielectric strength of the present experimental arrangement, when subnanosecond electric pulses are applied, is much higher compared to cases when $50-\mathrm{Hz}$ ac, microsecond or longer duration pulses are applied. The complete experimental configuration is presented in Fig. 2. An SMAtype V-dot probe is mounted just a few millimeters below the cuvette with the connector's body attached to the ground plate electrode. This probe is used to control the voltage across the cuvette, with the electric field in the cuvette obtained using the numerical technique described in the following.

\section{NumericAl TECHNiQues}

A detailed 3-D modeling and EM analysis were carried out for the PEF system. To shorten the time required for numerical analysis of the $50-\Omega$ (RG214-U) coaxial cable, only a length of $101 \mathrm{~mm}$ was considered, corresponding to a propagation time of $0.5 \mathrm{~ns}$. The 3-D CST model of the
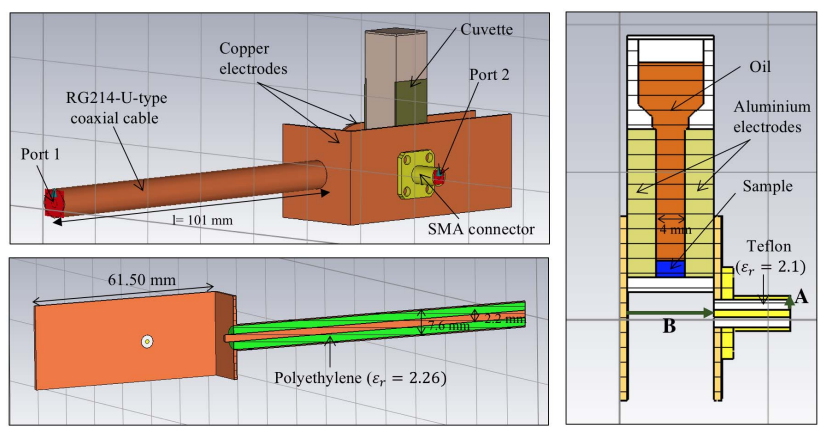

Fig. 3. Complete CST model for the PEF processing chamber, including the RG214-U coaxial cable, the two parallel-plate copper electrodes with the SMA-type V-dot probe attached and the electroporation cuvette, with various compartments filled with paraffin oil and water sample, which may contain bacteria. The two CST ports are highlighted.

PEF processing load is presented in Fig. 3. The presence of bacteria in the water contained inside the cuvette was not taken into consideration in the CST simulation, as measurements of electrical permittivity and the real signals recorded from the SMA-type V-dot probe, both demonstrated the bacteria do not introduce a significant difference. To analyze the impedance profile along the coaxial cable and in the PEF processing load and to better understand its behavior due to the presence of the electroporation cuvette, we have numerically performed the time domain reflectometry (TDR) task in CST. The TDR was evaluated based on the reflected signals using the integrated Gaussian signal shown in Fig. 4, with a rise time of 219 ps and a frequency bandwidth of $4 \mathrm{GHz}$, following the CST manual formula [30]:

$$
Z(t)=Z_{0} \frac{\int_{0}^{\infty} i(\tau) d \tau+\int_{0}^{t} o(\tau) d \tau}{\int_{0}^{\infty} i(\tau) d \tau-\int_{0}^{t} o(\tau) d \tau}
$$

where $Z_{0}$ is the characteristic impedance of the coaxial cable, i.e., $50 \Omega, i(t)$ is the incident signal at CST Port 1 (Fig. 3) and $o(t)$ is the reflected signal at the same port. Fig. 5 shows the impedance is $50 \Omega$ along the coaxial cable and then at $1 \mathrm{~ns}$, which corresponds to the return end of the coaxial cable, the impedance starts to increase to a value of $70 \Omega$. This effect is due to the mismatch between the coaxial cable and the PEF processing load. At around $1.2 \mathrm{~ns}$ the impedance starts to decrease and at $1.4 \mathrm{~ns}$ attains a minimum value of $32 \Omega$. This impedance decrease is due to the cuvette, which is placed at a distance of $121 \mathrm{~mm}$ from the coaxial cable input (Port 1). At around $1.8 \mathrm{~ns}$ the impedance again increases rapidly this time due to the open end of the PEF load. Both incident and reflected signals at Port 1 were carefully analyzed to observe the impedance distribution within the electric assembly. In addition, the estimation of the TDR allows us to have a representation of the time variation of the PEF processing load reflection coefficient (Fig. 6).

The electric field distribution inside the cuvette was studied using the CST Electrostatics solver. The cuvette behaves like a collection of two parallel plate capacitors mounted in parallel, one containing paraffin oil and the other water. Because of this, the electric field strength is the same in the two media, but the displacement fields are different. CST calculations indicate for 


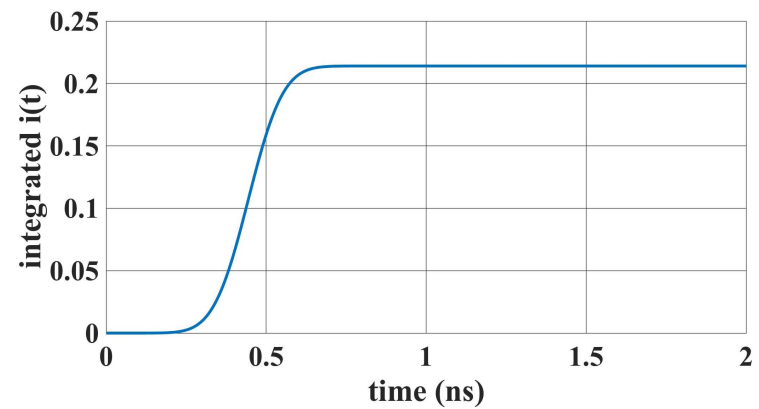

Fig. 4. Integrated Gaussian signal used to perform the TDR task.

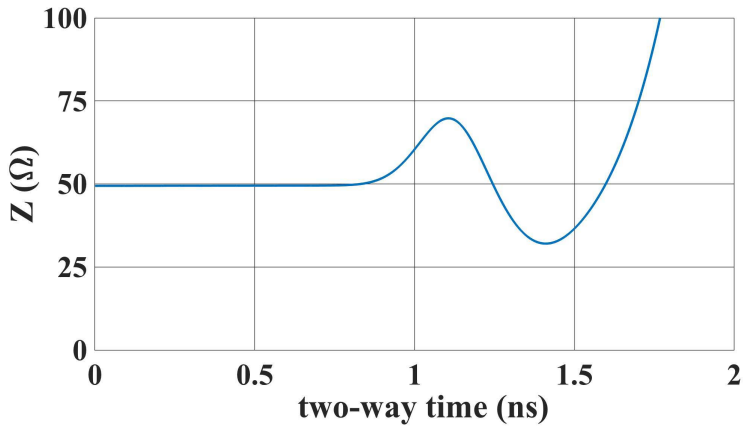

Fig. 5. Impedance profile along the cable and the processing load.

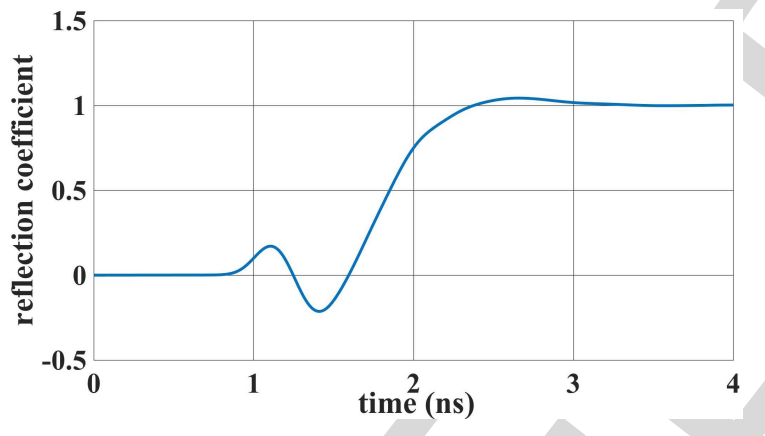

Fig. 6. Time variation of the reflection coefficient for the PEF processing load.

the cuvette a total equivalent capacitance of $5.95 \mathrm{pF}$, a value allowing voltage pulses having about 258-ps rise time to be delivered to the PEF processing load.

\section{Determination of the Time Variation of the Electric Field in the Processed Water SAMPle}

Because of the very small size of the water sample to be processed, there are no available techniques that can be used to directly measure the electric field generated inside. The only way to determine this essential parameter, which allows the correct interpretation of the biological results, is to use a combination of numerical modeling predictions and experimental evidence. Firstly, a detailed 3-D CST electromagnetic analysis of the load was carried out, including the SMA-type V-dot probe mounted near the cuvette (Fig. 7). The input signal applied to the processing load (see Fig. 8) was accurately measured using the main voltage probe attached to the RG214-U coaxial cable described above. This experimentally
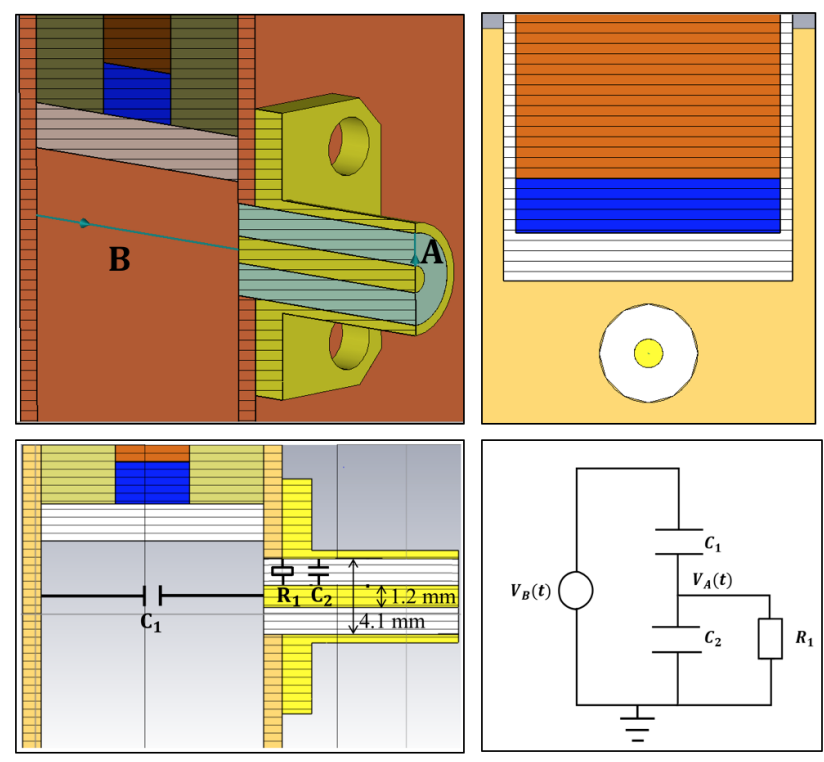

Fig. 7. Top: V-dot probe mounted inside the PEF load, near the cuvette containing the water to be processed. Bottom: its corresponding equivalent electric scheme, following that described in [32] and [33].

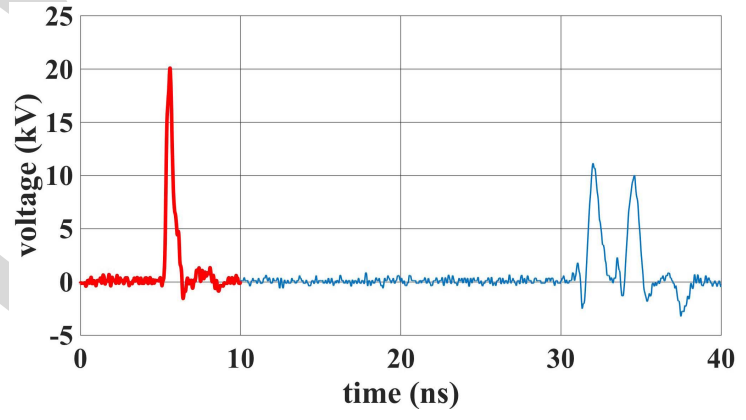

Fig. 8. Complete signal recorded from the main voltage probe considering the total length of $2.5 \mathrm{~m}$ for the RG214-U coaxial cable. The first $10 \mathrm{~ns}$ of the experimental voltage signal (highlighted) are used as CST input data.

obtained voltage signal, with a rise time of about $258 \mathrm{ps}$ and FWHM about $434 \mathrm{ps}$, was then used as an input (or excitation signal) for the CST Port 1 situated at the coaxial cable input (Fig. 3). To check the model, the predicted reflected signal was then successfully compared with the experimentally recorded reflected signal (see Fig. 9). To allow this comparison, the time delay of the signal due to the 2.5-m coaxial cable (see Fig. 8) was adjusted to the corresponding shorter time delay of only 101-mm length of cable considered by the CST model (see Fig. 9). A second comparison between CST predictions and the experimental data was performed for the CST Port 2 situated at the V-dot probe output (Fig. 3). Fig. 10 demonstrates the very good similarity between the predicted and the measured signal.

The above presented preliminary work had two important consequences.

1) Following [32], the SMA-type V-dot probe could be calibrated, with the voltage $V(t)$ calculated from the 


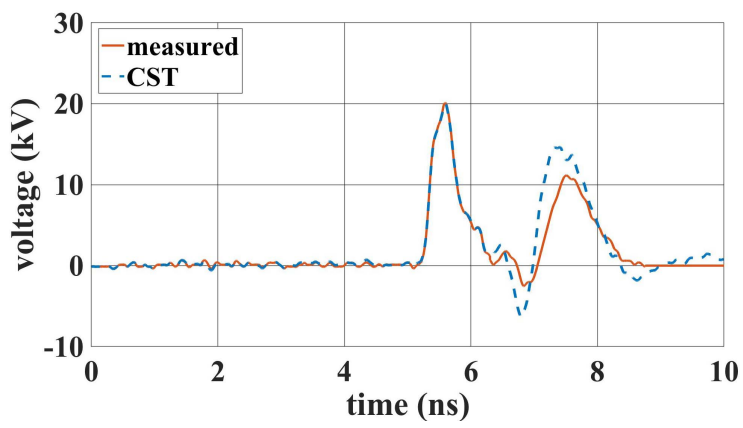

Fig. 9. Full line: experimental signal obtained from the main voltage probe. Dotted line: CST prediction for the voltage at Port 1 .

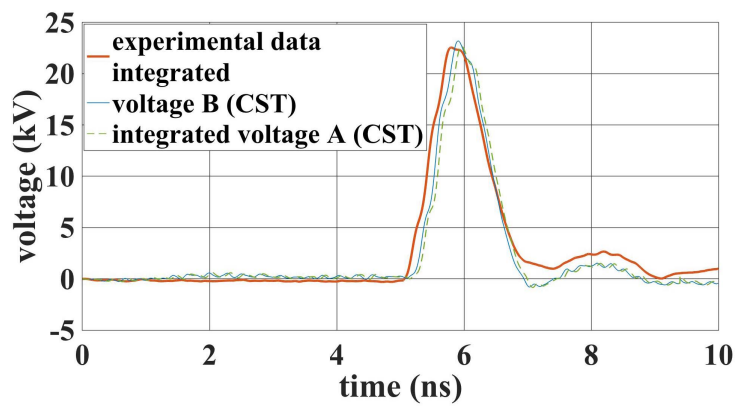

Fig. 10. Second comparison between CST model and experimental data. Thick line: time dependence of the integrated voltage signal from V-dot probe. Thin line: CST prediction obtained from a virtual voltage probe placed at location B. Dotted line: integrated voltage signal output from Port 2 placed at location A. The location of both A and B is presented in Fig. 7. The two CST generated traces are practically indistinguishable demonstrating that, due to the favorable electric field distribution, the SMA probe acts indeed as a V-dot probe [33].

probe output voltage signal $V_{\text {signal }}(t)$ as:

$$
V(t)=\frac{1}{\tau} \int_{0}^{t} V_{\text {signal }}(t) d t
$$

where $\tau=Z_{0} \cdot C_{1}$, with $Z_{0}=50 \Omega$ being the impedance of the cable connecting the probe to oscilloscope and $C_{1}=2.9 \mathrm{fF}$ the probe coupling capacitance [32], [33], as shown in Fig. 7 was obtained using CST calculations.

2) The following procedure was established. During testing, the electric field generated inside the water sample is calculated by CST, using an input voltage Port 1 based on the experimentally measured input voltage signal. However, as a precaution, the CST predictions for Port 2 are always checked against the experimental signal generated by the V-dot probe housed inside the load. If the two are identical, the CST results are most likely correct. If the two are different, a possible explanation could be that electric breakdowns are present inside the processing load.

As an example, Fig. 11 presents the calculated time variation of the electric field strength inside the water sample for the experimental conditions of Fig. 9, i.e., when the peak transient voltage input reaches a peak of $20 \mathrm{kV}$. The corresponding peak electric field strength reaches $58 \mathrm{kV} / \mathrm{cm}$, with a rise time of 303 ps and an FWHM of 773 ps. At this moment, the corresponding electric field distribution inside the processing load

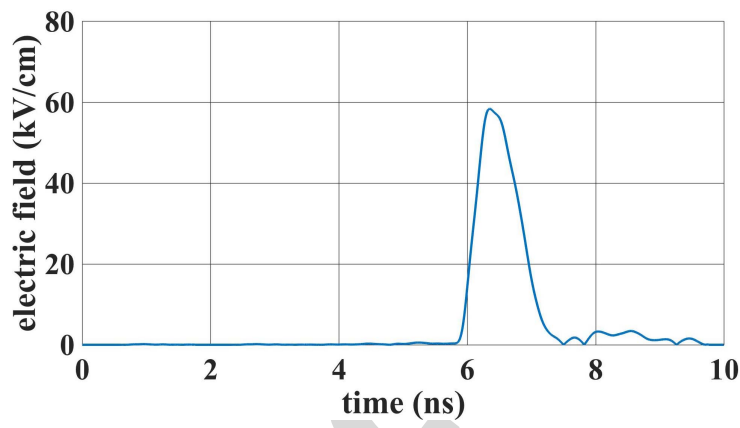

Fig. 11. PEF parameters generated inside the cuvette water sample, for the input conditions of Fig. 9. The peak electric field strength is reaching $58 \mathrm{kV} / \mathrm{cm}$, with a rise time of 303 ps and a FWHM of 773 ps.

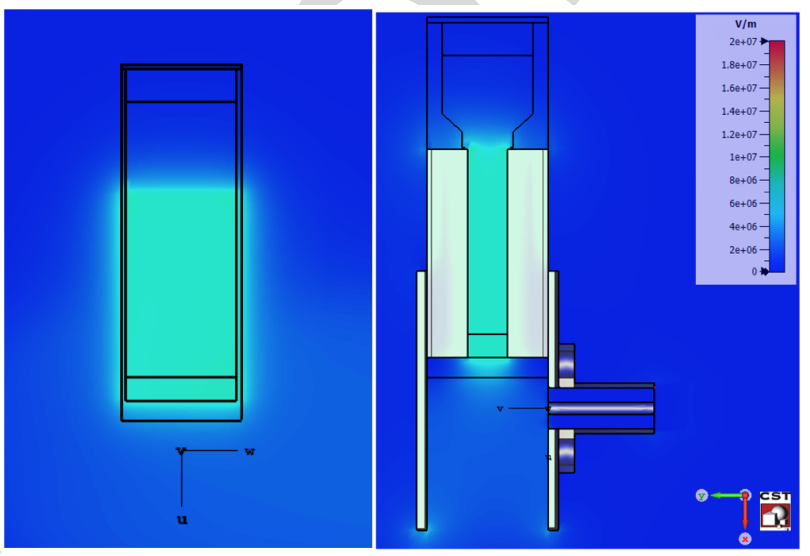

Fig. 12. Electric field distribution inside the processing load at the moment the peak field of $58 \mathrm{kV} / \mathrm{cm}$ is generated inside the cuvette water sample (see Fig. 11)

is shown in Fig. 12. It is important to note two essential characteristics: 1) the electric field is mainly concentrated inside the cuvette and 2) inside the cuvette the electric field is highly homogeneous, allowing a simple interpretation of the results obtained after PEF processing.

When performing a large number of tests at a high PRF, one important question is related to the possible temperature increase of the water sample. The variation of temperature $\Delta T$ during $N$ consecutive shots is given by

$$
\Delta T=\frac{W}{m C_{p}} N
$$

where $W$ is energy deposited in the water during one shot, $m \approx$ $100 \mathrm{mg}$ is the mass of the water sample and $C_{p}=4188 \mathrm{~J} / \mathrm{kg} / \mathrm{K}$ is the constant-pressure heat capacity of water at $25^{\circ} \mathrm{C}$ [34]. The energy is calculated as

$$
W=R \int_{0}^{t} I^{2}\left(t^{\prime}\right) d t^{\prime}
$$

where $R$ is the water sample resistance while the current integral was performed using data from a CST virtual current sensor. As the water sample has a conductivity of about $\sigma=300 \mu \mathrm{S} / \mathrm{m}$ and a relative permittivity $\varepsilon_{r}=78.4$ at $25{ }^{\circ} \mathrm{C}$ [35], the time constant $\varepsilon_{0} \varepsilon_{r} / \sigma=140 \mu \mathrm{s}$ is much larger than the pulse duration $(\approx 2 \mathrm{~ns})$ and therefore the conduction 
current can safely be ignored. In such conditions the water series equivalent resistance can be estimated as

$$
R=\frac{\tan \delta}{2 \pi f C_{w}}=1.28 \Omega
$$

where $\tan \delta=0.005$ [35] is the water loss tangent at a frequency $f$ estimated as $f=0.35 / t_{\text {rise }}=1.35 \mathrm{GHz}$, where $t_{\text {rise }}=258 \mathrm{ps}$ is the rise time of the impulse. The equivalent capacitance $C_{w}$ of the water sample was calculated using CST as $C_{w}=4.6 \mathrm{pF}$. As a typical result, after applying $N=50000$ consecutive shots, the estimated water sample temperature increase is only $\Delta T=0.00615^{\circ} \mathrm{C}$ and therefore negligible.

\section{Biological TeChNiques}

E. Coli DH5a were purchased from Invitrogen (Courtaboeuf, France). Cultures used for experiments were grown from $80 \mu \mathrm{L}$ of bacterial stock solution (saturated bacterial broth mixed with glycerol in $(1: 1)$ ratio stored at $-80{ }^{\circ} \mathrm{C}$ ) added to $8 \mathrm{~mL}$ of Luria Bertani (LB) medium (Invitrogen LB broth base Lennox L) and incubated at $37^{\circ} \mathrm{C}$ under constant orbital shaking at $220 \mathrm{r} / \mathrm{min}$ for $8 \mathrm{~h}$ over day, corresponding to an endpoint OD600 of approximately 0.9. Cultures were then placed at $4{ }^{\circ} \mathrm{C}$ overnight until used for experiments on the next day. E. Coli permeabilization was assessed using a cell-impermeant fluorescent nucleic acid stain entering only permeabilized bacteria, the YO-PRO-1 iodide ( $\lambda_{e x} 491 / \lambda_{e m}$ 509) (Invitrogen). E. Coli gating in flow cytometry was validated using a cell-permeant fluorescent nucleic acid stain entering all bacteria, permeabilized or not, the SYTO 9 ( $\left.\lambda_{e x} 485 / \lambda_{e m} 498\right)$ (Invitrogen). Prior to PEF exposure, bacteria were centrifuged at $2000 \times \mathrm{g}$ for $20 \mathrm{~min}$, the supernatant was discarded and the bacterial pellet was resuspended in the same volume of sterile deionized water (same bacterial concentration than mother culture). Then, either YO-PRO-1 iodide or SYTO 9 was added (working concentrations of 30 and $50 \mu \mathrm{M}$, respectively). A volume of $100 \mu \mathrm{L}$ of bacterial suspension with marker (YO-PRO-1 iodide or SYTO 9) was then placed in a 4-mm wide gap commercial electroporation cuvette (Cell Projects Ltd, Kent, U.K.) and covered with $1.6 \mathrm{~mL}$ of insulating paraffin oil in order to prevent arcing between electrodes during PEF exposure. Then, pulses were delivered at room temperature by installing the electroporation cuvette in the applicator presented in Fig. 3. Controls were sham exposures for which all the steps of the exposure protocol were followed, except for the PEF delivery. $5 \mathrm{~min}$ after PEF exposure, a volume of $50 \mu \mathrm{L}$ of the bacterial suspension was collected from the cuvette and mixed with $50 \mu \mathrm{L}$ of sterile deionized water. On average $30 \mathrm{~min}$ after PEF exposure, and prior to flow cytometry analyses, this bacterial suspension was
TABLE I

Results From PERMEABILIZATION EFFECT FOR VARIOUS Number of Pulses APPlied WHEN AN Electric Field of $58 \mathrm{kV} / \mathrm{Cm}$ Was APPLIED AT 200-Hz PRF

\begin{tabular}{|c|c|c|}
\hline $\begin{array}{c}\text { Number of } \\
\text { pulses applied }\end{array}$ & $\begin{array}{c}\text { Percentage of } \\
\text { permeabilized bacteria } \\
\text { (mean } \pm \text { standard deviation) }\end{array}$ & $\begin{array}{c}\text { Number of } \\
\text { replicates* }\end{array}$ \\
\hline 5000 & $44.4 \pm 9.3$ & 3 \\
\hline 10000 & $69.4 \pm 12.3$ & 6 \\
\hline 25000 & $81.0 \pm 7.4$ & 6 \\
\hline 50000 & $89.3 \pm 4.7$ & 6 \\
\hline
\end{tabular}

* The "number of replicates " is the number of times a given condition was tested on bacteria coming from different independant bacterial cultures.

mixed with $600 \mu \mathrm{L}$ of LB medium. Flow cytometry analyses were performed using a BD Accuri C6 Flow Cytometer (Bd Biosciences, le Pont-de-Claix, France). An excitation laser of $488 \mathrm{~nm}$ and the FL1 bandpass filter 533/30 were used to detect fluorescence of either YO-PRO-1 iodide and SYTO 9 (the reason why these markers could not be used simultaneously). For data processing, E. Coli were gated and separated from debris based on FSC-A and SSC-A morphological criteria. Nevertheless, a small proportion of events was not SYTO 9 positive within this gating, meaning that there was still debris. Thus, the specificity of the gating was calculated as (6) and (7), shown at the bottom of the page.

The specificity of the gating was evaluated for the different experimental conditions (Sham or PEF exposures), and was considered for further data processing. Finally, the percentage of permeabilized bacteria was calculated using YO-PRO-1 iodide following (7).

\section{Preliminary Results}

We assessed the ability of the subnanosecond pulses delivered by our system to induce electroporation on E. Coli DH5 $\alpha$, applying trains of 5000 to 50000 pulses at a PRF of $200 \mathrm{~Hz}$ at an electric field amplitude of $58 \mathrm{kV} / \mathrm{cm}$ (the top electric field amplitude deliverable with the present arrangement). All conditions tested efficiently generated electroporation of the bacteria to YO-PRO-1 iodide, a fluorescent nucleic acid stain and cell permeabilization marker of approximately $630 \mathrm{Da}$. Moreover, under these conditions, we observed an additive effect of the pulses, the percentage of permeabilized bacteria increasing from $44.4 \% \pm 9.3 \%$ to $89.3 \% \pm 4.7 \%$ for 5000 to 50000 pulses applied, respectively (Table I). Importantly, no increase of the temperature within the samples submitted to PEF treatment was noticed under the conditions tested in this study, in line with the estimated temperature rise presented above under Section IV. Thus, the permeabilization of $E$. Coli observed in response to PEF exposure performed in this study cannot be attributed to thermal effects.

$$
\begin{aligned}
\text { Gating Specificity }(\mathrm{GS}) & =\frac{\text { number of SYTO } ® 9 \text { positive events }}{\text { total number of events }} \\
\% \text { permeabilized bacteria } & =\frac{\frac{\% \text { of YO-PRO-1 positive events in sample }}{\text { GS sample }}-\frac{\% \text { of YO-PRO-1 positive events in control }}{\text { GS control }}}{100-\frac{\% \text { of YO-PRO-1 positive events in control }}{\text { GS control }}} \times 100
\end{aligned}
$$




\section{CONCLUSion And the WAY AheAD}

A relatively simple arrangement for PEF processing driven by a subnanosecond pulsed power generator has been developed and tested. The preliminary results show that subnanosecond processing can be highly efficient. The way ahead will require the development of a compact, high PRF and mobile subnanosecond 0.5-MV pulsed power generator that, when coupled with a prolate reflector, will allow biomedical experimentation at (or near) a hospital.

\section{ACKNOWLEDGMENT}

The authors would like to thank Valérie Bertrand and Edson Martinod from the Electrical Engineering Department, Xlim Research Institute, University of Limoges, France, for their help and discussion on CST simulations. They would also like to thank Anthony Ranchou-Peyruse from the Enviromental Microbiology Laboratory, IPREM, Université de Pau et des Pays de l'Adour (UPPA), France, for providing access to laboratory facilities.

\section{REFERENCES}

[1] R. P. J. K. H. Schoenbachk, "Ultrashort electrical pulses open a new gateway into biological cells," in Proc. Conf. Rec. 26th Int. Power Modulator Symp., High-Voltage Workshop., 0, pp. 205-209.

[2] D. Miklavčič, L. M. Mir, and P. T. Vernier, "Electroporation-based technologies and treatments," J. Membrane Biol., vol. 236, no. 1 pp. 1-2, Jul. 2010, doi: 10.1007/s00232-010-9287-9.

[3] E. Neumann, M. Schaefer-Ridder, Y. Wang, and P. H. Hofschneider, "Gene transfer into mouse lyoma cells by electroporation in high electric fields," EMBO J., vol. 1, no. 7, pp. 841-845, 1982. [Online]. Available: https://pubmed.ncbi.nlm.nih.gov/6329708

[4] M. J. Jaroszeski, R. Gilbert, and R. Heller, "Electrochemotherapy: An emerging drug delivery method for the treatment of cancer," Adv. Drug Del. Rev., vol. 26, nos. 2-3, pp. 185-197, Jul. 1997. [Online]. Available: http://www.sciencedirect.com/science/article/pii/S0169409X97000343

[5] T. Murakami and Y. Sunada, "Plasmid DNA gene therapy by electroporation: Principles and recent advances," Current Gene Therapy, vol. 11, no. 6, pp. 447-456, Dec. 2011. [Online]. Available: https://www.ingentaconnect.com/content/ben/cgt/2011/ 00000011/00000006/art00004

[6] D. Knorr, A. Angersbach, M. N. Eshtiaghi, V. Heinz, and D.-U. Lee, "Processing concepts based on high intensity electric field pulses," Trends Food Sci. Technol., vol. 12, nos. 3-4, pp. 129-135, Mar. 2001. [Online]. Available: http://www.sciencedirect.com/ science/article/pii/S0924224401000693

[7] B. Rubinsky, "Irreversible electroporation in medicine," Technol. Cancer Res. Treatment, vol. 6, no. 4, pp. 255-259, Aug. 2007, doi: $10.1177 / 153303460700600401$

[8] L. G. P. H. Vroomen, E. N. Petre, F. H. Cornelis, S. B. Solomon, and G. Srimathveeravalli, "Irreversible electroporation and thermal ablation of tumors in the liver, lung, kidney and bone: What are the differences?" Diagnostic Interventional Imag., vol. 98, no. 9, pp. 609-617, Sep. 2017.

[9] R. V. Davalos, B. Rubinsky, and L. M. Mir, "Theoretical analysis of the thermal effects during in vivo tissue electroporation," Bioelectrochemistry, vol. 61, nos. 1-2, pp. 99-107, Oct. 2003. [Online]. Available: http://www.sciencedirect.com/science/article/pii/S156753940300080X

[10] K. Nurgali, R. T. Jagoe, and R. Abalo, "Editorial: Adverse effects of cancer chemotherapy: Anything new to improve tolerance and reduce sequelae?" Frontiers Pharmacol., vol. 9, p. 245, Mar. 2018.

[11] J. C. Coffey, M. J. F. Smith, J. H. Wang, D. Bouchier-Hayes, T. G. Cotter, and H. P. Redmond, "Cancer surgery: Risks and opportunities," BioEssays, vol. 28, no. 4, pp. 433-437, Apr. 2006.

[12] C. E. Baum, "Focal waveform of a prolate-spheroidal impulse-radiating antenna," Radio Sci., vol. 42, no. 6, pp. 1-11, Dec. 2007.

[13] S. Altunc, C. E. Baum, C. G. Christodoulou, E. Schamiloglu, and C. J. Buchenauer, "Focal waveforms for various source waveforms driving a prolate-spheroidal impulse radiating antenna (IRA)," Radio Sci., vol. 43, no. 4, pp. n/a-n/a, Aug. 2008, doi: 10.1029/2007RS003775.
[14] S. Altunc, C. Baum, C. Buchenauer, C. Christodoulou, and E. Schamiloglu, "Design of a special dielectric lens for concentrating a subnanosecond electromagnetic pulse on a biological target," IEEE Trans. Dielectrics Electr. Insul., vol. 16, no. 5, pp. 1364-1375, Oct. 2009.

[15] S. Xiao, M. A. Migliaccio, J. C. Camp, J. F. Kolb, K. H. Schoenbach, and C. E. Baum, "A wide-band focusing system for bioelectric applications," in Proc. 16th IEEE Int. Pulsed Power Conf., Jun. 2007, pp. 744-747.

[16] R. A. Petrella, K. H. Schoenbach, and S. Xiao, "A dielectric rod antenna for picosecond pulse stimulation of neurological tissue," IEEE Trans. Plasma Sci., vol. 44, no. 4, pp. 708-714, Apr. 2016.

[17] F. Guo, C. Yao, C. Bajracharya, S. Polisetty, K. H. Schoenbach, and S. Xiao, "Simulation study of delivery of subnanosecond pulses to biological tissues with an impulse radiating antenna," Bioelectromagnetics, vol. 35, no. 2, pp. 145-159, Feb. 2014, doi: 10.1002/bem.21825.

[18] S. Xiao, S. Altunc, P. Kumar, C. E. Baum, and K. H. Schoenbach, "A reflector antenna for focusing subnanosecond pulses in the near field," IEEE Antennas Wireless Propag. Lett., vol. 9, pp. 12-15, 2010.

[19] P. Kumar et al., "A hyperband antenna to launch and focus fast highvoltage pulses onto biological targets," IEEE Trans. Microw. Theory Techn., vol. 59, no. 4, pp. 1090-1101, Apr. 2011.

[20] M. Croizer, "Applicators destined for the studies biological effects of electrical waves subnanoseconds," M.S. thesis, Univ. de Limoges, Limoges, France, Dec. 2015. [Online]. Available: https://tel.archivesouvertes.fr/tel-01298291

[21] K. H. Schoenbach et al., "The effect of intense subnanosecond electrical pulses on biological cells," IEEE Trans. Plasma Sci., vol. 36, no. 2, pp. 414-422, Apr. 2008.

[22] S. Xiao, S. Guo, V. V. Nesin, R. Heller, and K. H. Schoenbach, "Cell membrane permeabilization and death caused by subnanosecond electric pulses," in Proc. IEEE Int. Power Modulator High Voltage Conf., May 2010, pp. 194-199.

[23] I. Semenov, S. Xiao, D. Kang, K. H. Schoenbach, and A. G. Pakhomov, "Cell stimulation and calcium mobilization by picosecond electric pulses," Bioelectrochemistry, vol. 105, pp. 65-71, Oct. 2015.

[24] S. Xiao, S. Guo, V. Nesin, R. Heller, and K. H. Schoenbach, "Subnanosecond electric pulses cause membrane permeabilization and cell death," IEEE Trans. Biomed. Eng., vol. 58, no. 5, pp. 1239-1245, May 2011.

[25] J. T. Camp et al., "Cell death induced by subnanosecond pulsed electric fields at elevated temperatures," IEEE Trans. Plasma Sci., vol. 40, no. 10, pp. 2334-2347, Oct. 2012.

[26] Y.-Y. Hua, X.-S. Wang, Y. Zhang, C.-G. Yao, X.-M. Zhang, and Z.-A. Xiong, "Intense picosecond pulsed electric fields induce apoptosis through a mitochondrial-mediated pathway in HeLa cells," Mol. Med. Rep., vol. 5, no. 4, pp. 981-987, Apr. 2012. [Online]. Available: https://pubmed.ncbi.nlm.nih.gov/22307872

[27] J. Jia, Z.-A. Xiong, Q. Qin, C.-G. Yao, and X.-Z. Zhao, "Picosecond pulsed electric fields induce apoptosis in a cervical cancer xenograft," Mol. Med. Rep., vol. 11, no. 3, pp. 1623-1628, Mar. 2015. [Online]. Available: https://pubmed.ncbi.nlm.nih.gov/25405328

[28] B. M. Novac et al., "Demonstration of a novel pulsed electric field technique generating neither conduction currents nor Joule effects," IEEE Trans. Plasma Sci., vol. 42, no. 1, pp. 216-228, Jan. 2014.

[29] B. M. Novac, R. Xiao, P. Senior, L. Pecastaing, and I. R. Smith, "Generation of intense PEFs using a prolate spheroidal reflector attached to the bipolar former of a 10-GW pulsed power generator," IEEE Trans. Plasma Sci., vol. 46, no. 10, pp. 3547-3551, Oct. 2018.

[30] Computer Simulation Technology. Accessed: Apr. 28, 2020. [Online]. Available: https://www.3ds.com/

[31] L. Pécastaing, J. Paillol, T. Reess, A. Gibert, and P. Domens, "Design and performance of high voltage pulse generators for ultra-wideband applications," Meas. Sci. Technol., vol. 12, no. 10, pp. 1718-1725, Sep. 2001, doi: 10.10882F0957-02332F122F102F316.

[32] C. A. Ekdahl, "Voltage and current sensors for a high-density z-pinch experiment," Rev. Sci. Instrum., vol. 51, no. 12, pp. 1645-1648, 1980, doi: 10.1063/1.1136140.

[33] B. M. Novac et al., "Theoretical and experimental studies of Offthe-Shelf V-Dot probes," IEEE Trans. Plasma Sci., vol. 46, no. 8, pp. 2985-2992, Aug. 2018.

[34] M. W. Chase, NIST-JANAF Thermochemical Tables (Journal of Physical and Chemical Reference Data Monograph), 4th ed. Sep. 1998, pp. 1-1951.

[35] M. Zahn, Y. Ohki, D. B. Fenneman, R. J. Gripshover, and V. H. Gehman, "Dielectric properties of water and water/ethylene glycol mixtures for use in pulsed power system design," Proc. IEEE, vol. 74, no. 9, pp. 1182-1221, Sep. 1986. 


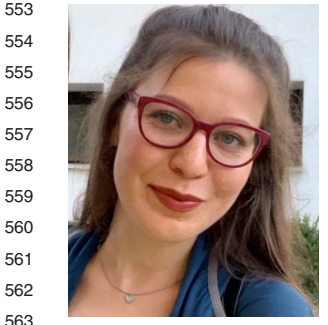

Njomza Ibrahimi (Graduate Student Member, IEEE) received the B.Sc. degree in electrical engineering from the University of Pristina, Pristina, Kosovo, in 2015, and the M.Sc. degree from the University of Pristina in 2017, combined with a one-year exchange study/research period at the Norwegian University of Science and Technology, Gjøvik, Norway. She is currently pursuing the Ph.D. degree with the Department of Electrical Engineering, Université de Pau et des Pays de l'Adour (UPPA), Pau, France.

Her research interests include high-voltage pulsed power systems and high-power microwave generation and transmission and their application in the biomedical domain.

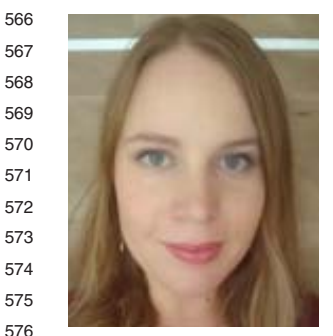

Leslie Vallet received the M.Sc. degree in chemical engineering from CPE Lyon Engineering School, Villeurbanne, France, in 2018, associated with one-year exchange study period at the Ecole Polytechnique Fédérale de Lausanne, Lausanne, Switzerland, specializing in biology. She is currently pursuing the Ph.D. degree with the METSY Unit, Gustave Roussy European Cancer Centre, Villejuif, France.

Her research interests include the interactions between biological cells and electric fields for medical application purposes.

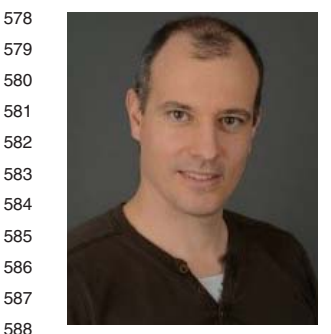

Franck M. Andre (Member, IEEE) received the master's degree in engineering (Science for Biological and Food Industries) from the Paris Institute of Technologies AgroParisTech (ex INA P-G), Paris, France, in 2002, and the Ph.D. degree for his research performed on in vivo gene electrotransfer from the ABIES Doctoral School, Paris Institute of Technologies AgroParisTech, in 2006.

During his postdoc (2007-2009) at the Frank Reidy Research Center for Bioelectrics, Old Dominion University, Norfolk, VA, USA, he investigated the effects of nanosecond pulsed electric fields on cell and nuclear membrane permeabilization. He is currently a Researcher with the French National Centre for Scientific Research (CNRS), Villejuif, France. For the last few years, his research has been mainly focused on electroporation-based gene transfer and drug delivery and on the use of electric pulses to manipulate the cytosolic calcium oscillations.

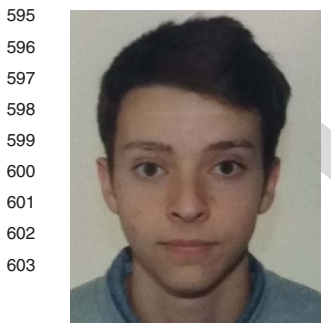

Laurent Ariztia received the B.Sc. and M.Sc. degrees from the Université de Pau et des Pays de l'Adour (UPPA), Pau, France, in 2017 and 2019, respectively.

$\mathrm{He}$ is currently an Engineer at the SIAME Laboratory. His research interests are mainly focused on pulsed power systems, such as Marx generators, and pulse forming lines for military and civil applications.

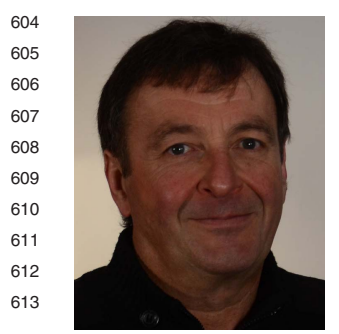

Marc Rivaletto was born in Tarbes, France, in 1960. $\mathrm{He}$ graduated from the Supelec Electrical Engineer School, Gif-sur-Yvette, France, in 1984, and the $\mathrm{Ph} . \mathrm{D}$. degree in electrical engineering from Pau University, Pau, France, in 1997.

$\mathrm{He}$ is currently a Lecturer at Pau University and works in the SIAME Laboratory. His research interests are HPM sources, compact pulsed power systems, including pulse forming lines, compact Marx generator, and resonant transformer.

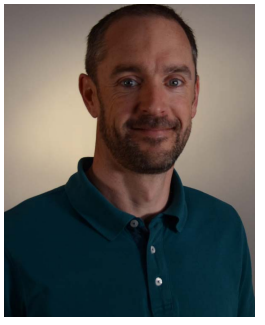

Antoine Silvestre de Ferron was born in Tarbes, France, in 1977. He received the master's degree in electrical and electronic engineering from the University of Toulouse, Toulouse, France, in 2002, and the Ph.D. degree in electrical engineering from the University of Pau, Pau, France, in 2006.

From 2006 to 2008, he was a Researcher with the Atomic Energy Commission (CEA), a French government-fund technological research organization in Le Barp, France. He is currently an Engineer with the Electrical Engineering Laboratory, University of Pau. His research interests include pulsed power generation, with military and civil applications and the high-voltage transient probes associated.

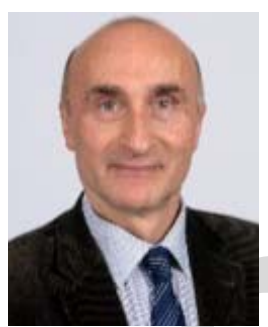

Bucur Mircea Novac (Senior Member, IEEE) received the M.Sc. and Ph.D. degrees from the University of Bucharest, Bucharest, Romania, in 1977 and 1989 , respectively.

He joined Loughborough University, Loughborough, U.K., in 1998, where he is currently a Professor of Pulsed Power. He has coauthored two books on explosive pulsed power and has published more than 200 refereed papers and conference contributions. His research interests include compact and repetitive high-power systems, explosively and electromagnetically driven magnetic flux compression generators and their applications, electromagnetic launchers, ultrafast magneto-optic and electrooptic sensors, and 2-D modeling of pulsed-power systems.

Dr. Novac is a Voting Member of the Pulsed Power Science and Technology Committee in the IEEE Nuclear and Plasma Science Society. He is also a member of the International Steering Committees for both the MEGAGAUSS Conferences and the Euro-Asian Pulsed Power Conferences. He is also the Co-Chairman of the U.K. Pulsed Power Symposium. He is a fellow of the Royal Academy of Engineering, and a Chartered Engineer and a fellow of the Institution of Engineering and Technology (IET), all in U.K.

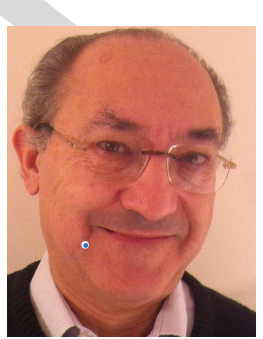

Lluis M. Mir graduated from École Normale Superieure, Paris, France, and received the D.Sc. degree from the University of Toulouse, Toulouse, France, in 1983, and three Doctorate Honoris Causa degrees from the Universities of Buenos Aires (Argentina), Lima (Peru), and Ljubljana (Slovenia), in 2018 .

As the DRCE at CNRS, he directed the Laboratory of Vectorology and Anticancer Therapies from 2010 to 2019 and the European Associated Laboratory for the applications of electric pulses in biology and medicine from 2011 to 2018 . He published 238 peer-reviewed articles and 25 book chapters $(\mathrm{H}$ index $=70$, WoS $)$.

Dr. Mir is a fellow of the American Institute for Medical and Biological Engineering, the European Academy of Tumor Immunology, and the RadioScience International Union (URSI). He won several awards, including the URSI's 2017 Balthasar van der Pol Gold Medal. He is the present President of the International Society for the Electroporation Based Treatments and Therapies.

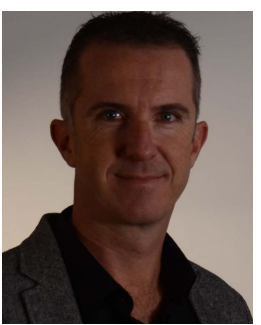

Laurent Pécastaing (Senior Member, IEEE) received the Ph.D. and Research Directorship Habilitation degrees in electrical engineering from the Université de Pau et des Pays de l'Adour (UPPA), Pau, France, in 2001 and 2010, respectively.

$\mathrm{He}$ is currently a Full Professor in pulsed power with the Laboratoire SIAME, UPPA. He is the Head of the High Voltage Processes Team, Laboratoire SIAME, and he is also the Director of a Common Laboratory, CEA, Paris, France. His current research interests include high-power microwave sources, compact pulsed power systems, and ultrafast transient probes.

Dr. Pécastaing is the Chairman of the next EAPPC/BEAMS/MEGAGAUSS Conference to be held in France in 2021. He is also a member of the International Steering Committees for both the BEAMS Conferences and the Euro-Asian Pulsed Power Conferences. 